\title{
Cancer screening in Australia: future directions in melanoma, Lynch syndrome, and liver, lung and prostate cancers
}

Marianne F Weber, ${ }^{a, b}$, Henry M Marshall ${ }^{c, d}$, Nicole Rankina ${ }^{a}$, Stephen Duffye, Kwun M Fong ${ }^{\mathrm{c}, \mathrm{d}}$, Kate Dunlop ${ }^{\mathrm{f}, \mathrm{g}}$, Lauren Humphreys ${ }^{\dagger}$, Amelia K Smit ${ }^{\dagger, h, i}$, Anne E Cust ${ }^{\dagger, i}$, Natalie Taylor ${ }^{a, j}$, Gillian Mitchell ${ }^{k, l}$, Yoon-Jung Kang ${ }^{a, j}$, Kathy Tucker ${ }^{m, n}$, Mark Jenkins ${ }^{\circ}$, Finlay Macrae $^{p, q}$, Ian Lockartr,s, Mark Dantar,s, Bruce K Armstrong ${ }^{\mathrm{b}, \mathrm{t}}$ and Megan Howe ${ }^{\mathrm{u}, \mathrm{v}}$

${ }^{a}$ Cancer Research Division, Cancer Council NSW, Sydney, Australia

b School of Public Health, University of Sydney, NSW, Australia

c Department of Thoracic Medicine, The Prince Charles Hospital, Brisbane, QLD, Australia

d University of Queensland Thoracic Research Centre, The Prince Charles Hospital, Brisbane, Australia

e Queen Mary University of London, UK

${ }^{\dagger}$ Cancer Epidemiology and Prevention Research, Sydney School of Public Health, Faculty of Medicine and Health, University of Sydney, NSW, Australia

g Centre for Genetics Education, NSW Health, Sydney, Australia h Sydney Health Ethics, Sydney School of Public Health, Faculty of Medicine and Health, University of Sydney, NSW, Australia Melanoma Institute Australia, University of Sydney, NSW

Faculty of Health Sciences, University of Sydney, NSW, Australia ${ }^{k}$ The Sir Peter MacCallum Department of Oncology, University of Melbourne, VIC, Australia
Parkville Familial Cancer Centre, Peter MacCallum Cancer Centre, Melbourne, VIC, Australia

m Hereditary Cancer Centre, Prince of Wales Hospital, Sydney, NSW, Australia

n Prince of Wales Clinical School, UNSW Sydney, Australia

- Melbourne School of Population and Global Health, University of Melbourne, VIC, Australia

p Colorectal Medicine and Genetics, The Royal Melbourne Hospital, VIC, Australia

a Department of Medicine, The Royal Melbourne Hospital, University of Melbourne, VIC, Australia

' St Vincent's Clinical School, Faculty of Medicine, UNSW Sydney, Australia

s Department of Gastroenterology and Hepatology, St Vincent's Hospital, Sydney, NSW, Australia

t School of Population Health, University of Western Australia, Perth

"Sax Institute, Sydney, NSW, Australia

v Corresponding author: megan.howe@saxinstitute.org.au

\section{Article history}

Publication date: July 2019

Citation: Weber MF, Marshall HM, Rankin N, Duffy S, Fong KM, Dunlop K, Humphreys L, Smit AK, Cust AE, Taylor N, Mitchell G, Kang Y-J, Tucker K, Jenkins M, Macrae F, Lockart I, Danta M, Armstrong BK, Howe M. Cancer screening in Australia: future directions in melanoma, Lynch syndrome, and liver, lung and prostate cancers. Public Health Res Pract. 2019;29(2):e2921910 https://doi.org/10.17061/phrp2921910

\section{Abstract}

While Australia now has well-established national screening programs for breast, bowel and cervical cancers, research continues into the feasibility of developing systematic screening programs for a number of other cancers. In this paper, experts in their fields provide perspectives on the current state of play and future directions for screening and surveillance for melanoma, Lynch syndrome, and liver, lung and prostate cancers in Australia. Although the evidence does not support population screening, there may be opportunities to prevent thousands of deaths through systematic approaches to the early detection of lung cancer and melanoma, testing for Lynch syndrome, and organised surveillance for hepatocellular carcinoma among individuals at high risk - guided by targeted research. The paper also looks at what impact new prostate specific antigen testing guidelines are having on screening for prostate cancer. 


\section{Key points}

- Development of a targeted screening program for those at high risk of lung cancer - Australia's largest cause of cancer mortality - could prevent thousands of deaths, especially among former smokers

- Shifting from the current opportunistic approach to melanoma screening to a national, risk-stratified program may be a promising way forward to reduce the heavy burden of the disease

- Lynch syndrome is a large but hidden genetic condition. Systematic testing for the syndrome in patients with colorectal cancer, as well as genomic developments, could help address underdiagnosis
- A better system of routine surveillance for hepatocellular carcinoma is needed among high-risk populations to reduce the growing incidence and mortality from the disease in Australia

- More thorough evaluation is needed of the impact of new Australian guidelines on prostate specific antigen testing for prostate cancer, with Medicare data suggesting the guidelines have only had a limited impact on practice

\section{Opportunities for lung cancer screening in Australia}

By Marianne F Weber, Henry M Marshall, Nicole Rankin, Stephen Duffy and Kwun M Fong

Despite Australia's many successes in tobacco control, lung cancer is the nation's largest cause of cancer mortality and will be a major health burden for many decades. The US National Lung Screening Trial (NLST) demonstrated that lung cancer screening of current and former high-risk smokers using low-dose computed tomography (LDCT) was a cost-effective strategy that significantly reduced lung cancer mortality by $20 \%$ (95\% confidence interval $[\mathrm{Cl}] 6.8 \%, 26.7 \%) .{ }^{1}$ A preliminary report on the Netherlands-Leuvens screening trial also demonstrated LDCT screening effectiveness, finding a $26 \%$ relative reduction in lung cancer mortality $(95 \% \mathrm{Cl}$ $9 \%, 41 \%$ ) among high-risk men. ${ }^{2}$

Although a number of organisations now recommend annual lung screening with LDCT using variations of the NLST eligibility criteria, there is no organised, nationwide lung screening program in any country. ${ }^{3}$ This is largely due to the significant implementation challenges of translating trial-based outcomes into a population-based setting, and the complexities of developing evidence based lung screening guidelines. Challenges include questions around the appropriate target population; the optimal screening interval; and effective risk communication, recruitment strategies, nodule management, management of incidental findings and harm minimisation. ${ }^{4}$

In 2015, the Australian Government Standing Committee on Screening recommended against lung cancer screening in Australia until there is greater clarity about these issues and a favourable cost-effectiveness evaluation in the Australian setting. ${ }^{5}$ Unlike Australia's existing cancer screening programs (for bowel, breast and cervical cancers), in which the target population can be identified in national administrative databases based on age and sex, lung cancer screening requires information about people's smoking history. This adds complexity because: 1) smoking history is not systematically documented in any population-wide database; and 2) high-risk smokers are overrepresented in hard-to-reach populations, including those in remote and low socio-economic areas, and Aboriginal and Torres Strait Islander people. ${ }^{6}$

The evidence to support an Australian-based lung screening framework has been strengthened based on data from two local studies. The Queensland Lung Cancer Screening Study (2007-2014) demonstrated the feasibility of applying a NLST-like screening protocol in Australia, and achieved similar rates of benefits and harms to the NLST. ${ }^{7}$ The International Lung Screen Trial (ILST $)^{8}$ commenced in 2016 in Australia and Canada and will contribute prospective data on: 1) use of a widely validated, individualised risk calculator to define screening eligibility by identifying those at high risk for lung cancer ${ }^{9}$; and 2) the use of a risk model to differentiate screendetected lung nodules with a high risk of malignancy from those likely to be benign. ${ }^{10}$ Combined use of these two risk prediction models is expected to reduce the falsepositive rate and increase the positive predictive value of screening. The ILST will also provide data on costs, health-related quality of life, participation rates, recruitment strategies, adjunct smoking cessation initiatives and incidental detection of other diseases. These outcomes will inform reliable health economic evaluations of lung cancer screening in Australia and internationally.

Screening efficiency is also expected to improve with the implementation of new computed tomography (CT) technologies. Radiomics uses high-throughput image analysis to extract quantitative CT features such as voxel intensity, texture and shape to gain insights into tumour phenotype and microenvironment, which reflect underlying pathophysiology. ${ }^{11}$ Combined with patient characteristics and correlated with clinical outcomes, these data can be used to estimate the malignant potential of a nodule and support decision making. Machine-read, quantitative image analysis may also overcome current limitations in the sensitivity and specificity of qualitative, human-read CT images. Computer-aided detection software is commercially available as either a first or second reader alongside a human radiologist; however, it is yet to be integrated into clinical practice because of variation in the performance of algorithms that arises from technical differences and data training sets. ${ }^{12}$ 
Overall, combined investment in tobacco control and lung cancer screening could see major reductions in the lung cancer burden, and could offset the growing costs of targeted and immunotherapies for lung cancer. As new data about lung cancer screening accrue, optimisation of prevention strategies, as well as trade-offs between drivers of screening efficiency and cost-effectiveness, can be informed by modelling and implementation studies. Overall, lung cancer screening is an opportunity to prevent thousands of lung cancer deaths, especially for former smokers who make up an increasing proportion of lung cancer cases.

\section{Emerging issues in melanoma screening}

By Kate Dunlop, Lauren Humphreys, Amelia K Smit and Anne E Cust

Melanoma accounts for approximately $10 \%$ of all new invasive cancer diagnoses in Australia each year, and there are just as many in situ as invasive melanomas diagnosed. ${ }^{13}$ Skin cancer, including melanoma and keratinocyte cancers (i.e. non-melanoma skin cancer, predominantly basal cell and squamous cell carcinomas) are the most expensive cancer for the Australian healthcare system. ${ }^{14}$ Annual Australian health system costs for melanoma have increased from \$30 million in 2001 to \$201 million in 2014, and costs are growing exponentially with the expansion of new therapies for late-stage disease. ${ }^{15}$ Total (direct) treatment costs for keratinocyte cancers are almost 10 times that of melanoma, due to the sheer number diagnosed ${ }^{16}$, and treatments for suspicious but ultimately benign lesions add further to costs. ${ }^{15,17}$ This is signficant when considering population screening for melanoma, because, although keratinocyte cancers seldom cause death, they may add significantly to the costs of any screening program unless there are clear criteria in place to minimise the number of unnecessary excisions.

Early detection of melanoma is important as the prognosis is worse and the extent and costs of treatment are much higher for late-stage diagnoses. However, population screening for the early detection of melanoma is not recommended. ${ }^{18}$ While melanoma deaths are the result of metastatic disease, and early-stage melanoma can be visible without invasive procedures, there is insufficient evidence that population screening for melanoma using current technology and approaches can reduce mortality.

Notwithstanding this lack of evidence, opportunistic screening is common. The Royal Australian College of General Practitioners guidelines currently recommend opportunistic skin checks for people at moderately elevated risk, and skin checks every 6-12 months plus advice on skin self-examination for those at high risk. ${ }^{19}$ Cancer Council guidelines recommend that people at very high risk have 6-monthly skin checks supported by total body photography, dermoscopy and advice on skin self-examination. ${ }^{20}$

Despite the lack of an evidence base either for or against population-based screening, early detection of melanoma through a national population-based screening program has the potential to save lives, reduce morbidity and treatment-related costs, and encourage sun protection behaviours. Conversely, it may not be costeffective and might lead to unacceptable overdiagnosis and overtreatment of in situ and thin melanomas and keratinocyte cancers. So, is there a way through this impasse?

Development and evaluation of a risk-stratified approach to screening for melanomas is a promising way forward. This approach would involve screening schedules (detailing ages at which screening starts and stops, frequency of skin examinations and the type of technology used) being tailored to individual risk, based on a comprehensive assessment taking into account multiple risk factors. Such an approach aligns with the US Preventive Services Task Force recommendations that future research on skin cancer screening should focus on evaluating the effectiveness of targeted screening in those considered to be at higher risk. ${ }^{18} \mathrm{~A}$ risk-stratified approach could help maximise the benefits and minimise both the harms and health system costs of screening. ${ }^{21}$

New technologies such as surveillance photography, teledermatology, artificial intelligence, and apps to support patient self-examination and triage of clinical examinations also have the potential to reduce overtreatment or unnecessary treatment of some lesions detected in a screening program. These technologies could also improve equity of access to dermatological services for people living in regional and remote areas. ${ }^{22}$

Changing the paradigm of melanoma screening in Australia from an opportunistic approach to a national risk-stratified program would bring challenges. Such a change would require high-quality evidence about the benefits, harms and resource implications of a new program, as well as its feasibility and acceptability to the public and health professionals. It would require shifting health service resources away from screening low-risk patients towards higher-risk patients. Validated risk prediction tools, including online risk calculators, are now available to accurately estimate melanoma risk, but would need to be rolled out on a national scale. A Melanoma Screening Summit, hosted by the Australian Skin and Skin Cancer Research Centre in March 2019²3, demonstrated a collective intent to address these issues, and identified research priorities for evaluation of the evidence to optimise early detection of melanoma. 


\section{Opportunities for Lynch syndrome testing}

By Natalie Taylor, Gillian Mitchell, Yoon-Jung Kang, Kathy Tucker, Mark Jenkins and Finlay Macrae

Lynch syndrome is caused by an inherited pathogenic mutation in one of four mismatch repair genes $(M L H 1$, MSH2, MSH6, PMS2) and EPCAM. ${ }^{24}$ Mutations in these genes cause an increased lifetime risk of cancers, particularly colorectal cancer and endometrial cancer, often at a younger age than in the general population. Identification of Lynch syndrome carriers can save lives, as it enables prevention and early detection of cancers and cascade predictive testing for at-risk relatives ${ }^{25}$; this improves opportunities for better disease outcomes and lower long-term healthcare costs.

Up to $3 \%$ of colorectal and endometrial cancers have been attributed to Lynch syndrome, increasing to 7-10\% of those diagnosed before age 50. ${ }^{25}$ Germline gene testing in patients with colorectal cancer, particularly those with disease family history or with early age diagnosis, has been the most common approach to identifying Lynch syndrome. However, expanding this to testing in all incident colorectal cancer cases through initial tumour testing followed by germline genetic testing for mismatch repair deficient (dMMR) tumours has been encouraged. ${ }^{26}$ Currently, there is no consistent Australian national approach to colorectal or endometrial cancer tumour testing for Lynch syndrome, resulting in underdiagnosis of the syndrome nationally.

One in 280 people carry a mutation in a Lynch syndrome gene ${ }^{27}$, with an estimated 100,000 carriers in Australia. A 2017 report suggests approximately only $5 \%$ of Lynch syndrome carriers have been identified ${ }^{28}$, making it a large but hidden problem. Although population-level screening would not meet the World Health Organization screening criteria because of the prevalence of Lynch syndrome, there have been arguments for such screening for breast cancer gene mutations (including BRCA1 and BRCA2), which have a similar carrier frequency, given the high risk of disease. ${ }^{29}$

Internationally, progress has been made. Health technology assessments have generated enough support to initiate policy change and implementation of systematic testing for Lynch syndrome in all people diagnosed with colorectal cancer in England. ${ }^{30}$ In Australia, a comprehensive evaluation of Lynch syndrome testing in colorectal cancer cases found that initial tumour testing for MMR deficiency (dMMR) in incident colorectal cancer without any age restriction would be cost-effective at the indicative willingness-to-pay threshold of $A \$ 30,000-$ A $\$ 50,000$ per life-year saved. ${ }^{31}$
While this new knowledge could and should bolster support for the implementation of systematic testing for Lynch syndrome in colorectal cancer in Australia, the scientific landscape of genomics is moving at a pace which offers further opportunities for testing. For example, universal germline gene panel testing can potentially be cost-effective at the aforementioned indicative willingness-to-pay threshold, if testing is limited to colorectal cancer cases in those aged $<70$ years, compared with no testing. However, this is not currently cost-effective when compared with initial dMMR tumour testing approaches, either age restricted or universal. As the cost of gene panel testing is likely to reduce substantially over time, its cost-effectiveness will improve. ${ }^{31}$ Furthermore, genomic advances are likely to enable Lynch syndrome tumour sequencing to be used to guide personalised treatment decisions, which will provide clues to inform both the need for and the interpretation of germline testing.

These are all attractive prospects for identifying Lynch syndrome carriers. The reality for the complex adaptive health system, however, is fraught with challenges. Even current approaches are not straightforward - globally, non-standardised clinical and laboratory approaches, healthcare and public health system perspectives, and behavioural, social and communication factors have led to many missed opportunities to identify Lynch syndrome carriers. Despite policy changes in England, implementing systematic testing for the syndrome in colorectal cancer has faced practical and financial challenges. ${ }^{32}$

Although there would be clear benefits of universal genetic testing for all colorectal cancers, the likelihood is that clinical responsibilities which typically sit with genetic specialist services will be deployed to non-specialist clinicians (i.e. mainstreaming of test requests, referrals and genetic counselling). This has been the case with recent policy changes for $B R C A 1$ and $B R C A 2$ gene panel testing, which has presented its own unique set of challenges. ${ }^{33}$

Further, as the clinical phenotype of Lynch syndrome includes other tumour types such as endometrial cancer, a systematic approach to identifying carriers may, depending on cost-effectiveness analysis findings, need to be extended beyond the narrow colorectal cancer setting. Crucially, Australia should learn from experiences of Lynch syndrome testing in other jurisdictions to design appropriate strategies to support healthcare professionals. These lessons can also help prepare the health system to be ahead of the implementation curve for the mainstreaming of genomic advances on the horizon. 


\section{How can we improve}

\section{hepatocellular carcinoma surveillance?}

\section{By lan Lockart and Mark Danta}

The incidence and mortality of hepatocellular carcinoma (HCC) continues to rise in Australia ${ }^{34,35}$, with a doubling in age-standardised mortality rates in the past 15 years Most international liver societies recommend 6-monthly ultrasound-based surveillance of at-risk populations ${ }^{36}$, however, the data supporting its impact remain weak. A nationwide screening program is not recommended; instead, individuals at risk need to be identified and enrolled in surveillance.

There are two easily identified high-risk populations: all individuals with cirrhosis, irrespective of the aetiology; and subgroups with chronic hepatitis B. Individuals with chronic hepatitis $B$ require further stratification as they are at risk of HCC development even in the absence of cirrhosis. ${ }^{36}$

The evidence supporting surveillance among at-risk populations is largely derived from observational studies, which are subject to multiple biases. A meta-analysis of 47 studies demonstrated that surveillance was associated with improved early tumour detection, curative treatment eligibility and 3-year survival. ${ }^{37}$ Most studies had inherent limitations of retrospective observational studies, including selection, lead-time and length-time biases. A recent analysis of the Surveillance, Epidemiology and End Results (SEER)-Medicare database in the US found that surveillance continued to be associated with significantly higher 3-year survival after adjusting for leadtime and length-time biases. ${ }^{38}$ Studies from Italy ${ }^{39}$ and the Netherlands ${ }^{40}$ have also demonstrated a survival benefit after adjusting for lead-time bias.

The impact of surveillance on mortality is less clear. Two randomised controlled trials have been conducted, both more than 20 years ago, among large populations of Chinese individuals with chronic hepatitis B. One demonstrated a $37 \%$ reduction in mortality for those undergoing ultrasound-based surveillance ${ }^{41}$; however, the methodology has been widely criticised and the results may not apply to other at-risk populations in the current era. The other found that 6-monthly alphafetoprotein (AFP) testing alone did not improve mortality. ${ }^{42}$ In a recent case-control study using national data from the US Veterans Affairs healthcare system, a cohort of patients dying from HCC was compared with a wellmatched cirrhotic cohort. The analysis revealed that the frequency of ultrasound and AFP measurement over the 4-year study period was similar between cases and controls, suggesting no mortality benefit from surveillance. ${ }^{43}$ However, it is unclear if these results reflect limitations in the efficacy of surveillance or the low compliance with regular surveillance in clinical practice.
Patients in the HCC surveillance group only received intermittent ultrasounds performed at intervals of longer than 6 months. Another recent community-based trial among patients with positive chronic hepatitis B in China found that participation in ultrasound-based surveillance improved early tumour detection and survival but did not reduce HCC-related mortality. ${ }^{44}$

Although a clear mortality benefit has not been demonstrated, routine HCC surveillance generally fulfils criteria for a successful screening program: there are two easily identified populations that are at high risk; the tumour usually has a long latent preclinical phase detectable with imaging, and curative treatments are feasible if diagnosed at an early stage; and surveillance with ultrasound is an acceptable, low-risk technique with an overall sensitivity of $84 \%$ for all HCCs and $47 \%$ for early HCC, as demonstrated by meta-analysis. ${ }^{45}$

The reason surveillance has not had a clear impact on HCC mortality is complex. First, it may relate to suboptimal performance of available modalities. The sensitivity of ultrasound is relatively low for early HCC, and diagnostic accuracy is influenced by operators' expertise and patient factors such as metabolic syndrome (obesity and nonalcoholic fatty liver disease) and nodular liver disease. Other techniques such as contrast-enhanced ultrasound and magnetic resonance imaging may become more appropriate in these settings. The population may also be too broad, and higher-risk groups could be identified using age, gender, aetiology, fibrosis stage and new specific molecular biomarkers. ${ }^{46}$ More important, however, is the low rate of participation in regular surveillance of the at-risk population. In a population-based cohort of patients diagnosed with HCC in Australia, only 40\% participated in regular surveillance. ${ }^{47}$ In a community health centre in Victoria, only $27 \%$ of patients with chronic hepatitis B had optimal adherence to surveillance. ${ }^{48} \mathrm{~A}$ recent US study explored interventions aimed at improving ultrasound surveillance for HCC. Compared with no intervention, uptake was significantly increased by mailed outreach, both with and without the addition of patient navigation, which assessed barriers to surveillance and encouraged surveillance participation (23\% uptake among the outreach/navigation group, $18 \%$ in the outreach only group and $7 \%$ in the control group). ${ }^{49}$ Although these participation rates are still woefully inadequate, the study is one of the first to explore interventions to improve surveillance.

In Australia, we can do better. Although a mortality benefit has not been clearly demonstrated, regular ultrasound-based surveillance of at-risk individuals is associated with improved early tumour detection, eligibility for curative treatment and survival. Interventions that increase participation in HCC surveillance among at-risk individuals are needed in Australia to improve outcomes for this highly fatal and high-priority cancer. 


\section{Have the Australian PSA testing guidelines changed practice?}

\section{By Bruce K Armstrong}

First 'outed' for public consultation on 4 December 2014 at the World Cancer Congress in Melbourne, Australia's clinical practice guidelines, PSA testing and early management of test-detected prostate cancer, were formally approved by the National Health and Medical Research Council (NHMRC) on 2 November 2015, and ultimately released publicly on 20 January 2016. The guidelines have been available since through the NHMRC, Prostate Cancer Foundation of Australia and Cancer Council Australia ${ }^{50}$ websites. They cover the territory from informed choice to have prostate specific antigen (PSA) testing, through to testing itself and investigation of abnormal test results, to conservative management of prostate cancer (active surveillance and watchful waiting). A summary is also available.

With respect to PSA testing specifically, the guidelines recommend: "For men at average risk of prostate cancer who have been informed of the benefits and harms of testing and who decide to undergo regular testing for prostate cancer, offer PSA testing every 2 years from age 50 to age 69, and offer further investigation if total PSA is greater than $3.0 \mathrm{ng} / \mathrm{mL}$."

The Royal Australian College of General Practitioners is the most important professional body in terms of acceptance of this recommendation. It refers to the substance of the PSA guidelines in its Guidelines for preventive activities in general practice (known as 'The Red Book') $)^{19}$ in these terms: "Screening of asymptomatic (low risk) men for prostate cancer by prostate specific antigen (PSA) testing is not recommended because the benefits have not clearly been shown to outweigh the harms. ... Therefore, GPs have no obligation to offer prostate cancer screening to asymptomatic men. ... If after an informed process, perhaps using a decision aid, a man still requests prostate cancer screening, a PSA blood test is acceptable." Given the high degree of uncertainty about PSA testing among Australian GPs ${ }^{51}$, this statement could discourage general practitioners' uptake of the PSA testing recommendation.

What evidence is there that publication of the PSA guidelines' recommendation quoted above has made any difference with respect to PSA testing? As there is no formal evaluation of the guidelines, analysis of routinely collected health data is the best way to answer this question. Figure 1 summarises data collected by Medicare on benefits paid for PSA tests done at intervals of not less than 1 year, and not for monitoring of previously diagnosed prostatic disease. Given the guidelines' release history, any change in testing levels prompted by the recommendation would not be expected before the beginning of 2016 .
Figure 1. Trends in PSA tests (MBS Item number 66655) bulk-billed or reimbursed by Medicare, 2008-09 to 2017-18, by age

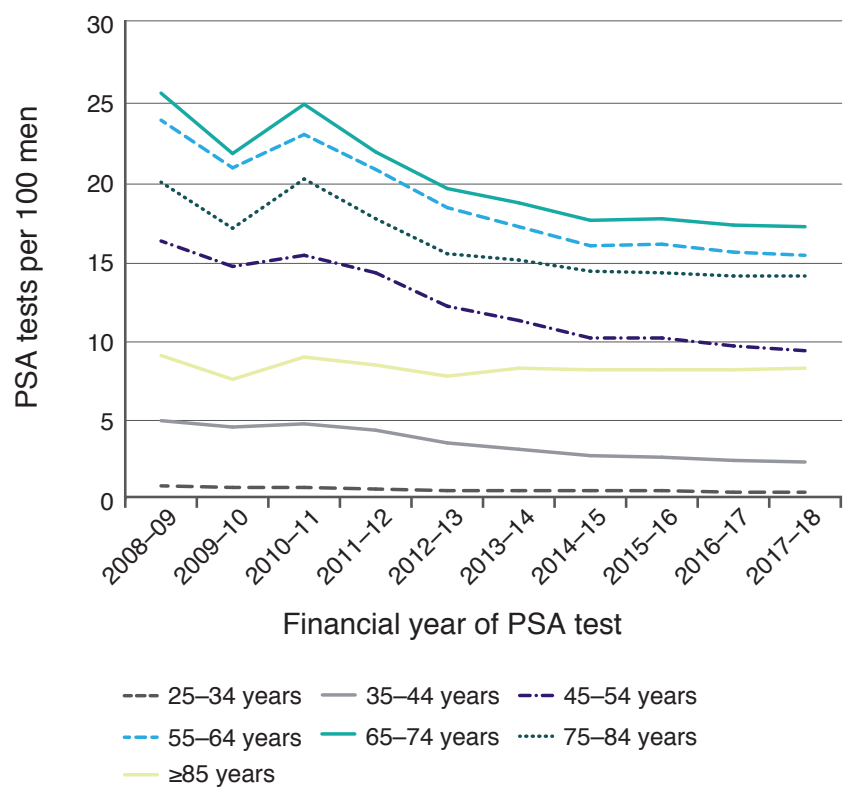

MBS = Medicare Benefits Schedule

Overall, after having fallen steadily since 2010-11 and reaching a plateau in 2014-15 and 2015-16, the rate of PSA testing fell a little in 2016-17 and 2017-18 in all age groups shown, except in men aged $25-34$ and $\geq 85$ years. These recent downtrends may indicate that the guidelines have had an effect in encouraging less PSA testing (perhaps by moving from annual testing to biennial testing as recommended). However, the lack of any reduction in testing rates in the $25-34$ and $\geq 85$ years age groups suggests that recommending first testing at 50 years and last testing at 69 years has not influenced practice. While prostate cancer incidence rates might also reflect such trends (through less overdiagnosis), the necessary data are not yet available nationally beyond 2014 .

It is probably not yet too late to plan and implement a more thorough evaluation of the impact of the PSA testing guidelines. Although rarely done, such an evaluation can guard against unanticipated harm that an ill-judged clinical guideline can produce, or can call to account poor implementation of a guideline with potential for benefit. ${ }^{52}$ 


\section{Acknowledgements}

AS received a Research Training Program (RTP) Stipend Scholarship and a Merit Top Up Scholarship from the University of Sydney, and Top Up Scholar Awards from the Sydney Catalyst Translational Cancer Research Centre and the Melanoma Institute Australia. AC received Career Development Fellowships from the NHMRC (1147843) and Cancer Institute NSW (15/CDF/1-14), and project grant funding from the NHMRC (1165936). NT has a fellowship from Cancer Institute NSW. MJ's institution receives grants from National Institutes of Health and NHMRC. GM received a grant from Cancer Council NSW to support Lynch syndrome research. FM's institution has received grants from the Cass Foundation and Cancer Council NSW, and he has grants pending from the National Institutes of Health.

\section{Peer review and provenance}

Externally peer reviewed, commissioned.

\section{Competing interests}

FM has done paid consultancy work for Rhythm Biosciences and received payment for providing medicolegal opinions. BA's then-employer, the Sax Institute, was reimbursed by the Prostate Cancer Foundation of Australia (PCFA) for remunerated time spent advising the systematic evidence review team for the PSA testing guideline and on writing parts of the guideline document. BA is a member of the PCFA's Board and Chair of their Research Advisory Committee. He receives no remuneration for either of these positions, but all expenses for attending meetings are paid for by the PCFA.

\section{Author contributions}

MW, HM, NR, SD and KF authored the perspective on lung cancer screening. $\mathrm{MW}$ and $\mathrm{HM}$ drafted the manuscript. NR, SD and KF provided comments on the fully drafted versions of the manuscript. The corresponding author for this section is $\mathrm{MW}$ : mariannew@nswcc.org.au

$\mathrm{KD}, \mathrm{LH}, \mathrm{AS}$ and $\mathrm{AC}$ authored the perspective on melanoma screening. KD, LH, AS and AC contributed equally to the preparation, writing and editing. The corresponding author for this section is $A C$ : anne.cust@sydney.edu.au

NT, GM, Y-JK, KT, MJ and FM authored the perspective on Lynch syndrome testing. NT drafted the manuscript and led the editorial process. GM, Y-JK, KT, MJ and FM contributed edits in the form of content knowledge and expertise to the manuscript. The corresponding author for this section is NT: natalie.taylor@nswcc.org.au
$\mathrm{IL}$ and MD authored the perspective on hepatocellular carcinoma surveillance. Both authors contributed to writing the manuscript and reviewed and approved the final version. The corresponding author for this section is MD:m.danta@unsw.edu.au

BA is the sole and corresponding author of the perspective on PSA testing: bruce@brucekarmstrong.org

$\mathrm{MH}$ wrote the abstract and key points, and collated and edited the final manuscript.

\section{References}

1. Black WC, Gareen IF, Soneji SS, Sicks JD, Keeler EB, Aberle DR, et al. Cost-effectiveness of CT screening in the National Lung Screening Trial. N Engl J Med. 2014;371:1793-802.

2. de Koning $\mathrm{H}$, Van Der Aalst $\mathrm{C}$, ten Haaf K, Oudkerk M, editors. Effects of volume CT lung cancer screening: mortality results of the NELSON Randomised-Controlled Population Based Trial. International Association for the Study of Lung Cancer 19th World Conference on Lung Cancer; 2018; Toronto, Canada.

3. Pinsky PF. Lung cancer screening with low-dose CT: a world-wide view. Translational Lung Cancer Research. 2018;7:234-42.

4. Moffat J, Hiom S, Kumar HS, Baldwin DR. Lung cancer screening - gaining consensus on next steps proceedings of a closed workshop in the UK. Lung Cancer. 2018;125:121-7.

5. Australian Government Department of Health. Standing Committee on Screening: lung cancer screening. Canberra: Australian Government Department of Health; 2015 [cited 2019 May 29]. Available from: www.cancerscreening.gov.au/internet/screening/ publishing.nsf/Content/lung-cancer-screening

6. Australian Institute of Health and Welfare. National Drug Strategy Household Survey 2016: detailed findings. Canberra: AlHW; 2017 [cited 2019 May 29]. Available from: www.aihw.gov.au/getmedia/15db8c15-7062-4cdebfa4-3c2079f30af3/21028a.pdf.aspx?inline=true

7. Marshall HM, Bowman RV, Ayres J, Crossin J, Lau M, Slaughter RE, et al. Lung cancer screening feasibility in Australia. Eur Respir J. 2015;45:1734-7.

8. National Institutes of Health, US National Library of Medicine: ClinicalTrials.gov. Bethesda, MD: NIH NLM; 2019. International Lung Screen Trial (ILST) (NCT02781856); 2016 [cited 2019 Jun 11]; [about 4 screens]. Available from: clinicaltrials.gov/ct2/show/NC T02871856? $\mathrm{id}=$ NCT02871856\&rank $=1$

9. Tammemagi MC, Katki HA, Hocking WG, Church TR, Caporaso N, Kvale PA, et al. Selection criteria for lungcancer screening. N Engl J Med. 2013;368:728-36. 
10. McWilliams A, Tammemagi MC, Mayo JR, Roberts $H$, Liu G, Soghrati K, et al. Probability of cancer in pulmonary nodules detected on first screening CT. N Engl J Med. 2013;369:910-9.

11 Thawani R, McLane M, Beig N, Ghose S, Prasanna P. Velcheti $\mathrm{V}$, et al. Radiomics and radiogenomics in lung cancer: a review for the clinician. Lung Cancer. 2018;115:34-41.

12. Silva M, Milanese G, Seletti V, Ariani A, Sverzellati N. Pulmonary quantitative CT imaging in focal and diffuse disease: current research and clinical applications. Br J Radiol. 2018;91:20170644.

13. Australian Institute of Health and Welfare. Cancer in Australia 2017. Canberra: AIHW; 2017 [cited 2019 May 21]. Available from: www.aihw. gov.au/getmedia/3da1f3c2-30f0-4475-8aed1f19f8e16d48/20066-cancer-2017.pdf.aspx?inline=true

14. Fransen M, Karahalios A, Sharma N, English DR, Giles GG, Sinclair RD. Non-melanoma skin cancer in Australia. Med J Aust. 2012;197(10):565-8.

15. Elliott TM, Whiteman DC, Olsen CM, Gordon LG. Estimated healthcare costs of melanoma in Australia over 3 years post-diagnosis. Appl Health Econ Health Policy. 2017;15:805-16.

16. Doran CM, Ling R, Byrnes J, et al. Estimating the economic costs of skin cancer in New South Wales, Australia. BMC Public Health. 2015;15:952.

17. Watts CG, Cust AE, Menzies SW, Mann GJ, Morton RL. Cost-effectiveness of skin surveillance through a specialized clinic for patients at high risk of melanoma. J Clin Oncol. 2017;35:63-71.

18. Wernli KJ, Henrikson NB, Morrison CC, Nguyen M, Pocobelli G, Blasi PR. Screening for skin cancer in adults: updated evidence report and systematic review for the US Preventive Services Task Force. JAMA. 2016;316:436-47.

19. The Royal Australian College of General Practitioners. Guidelines for preventive activities in general practice, 9th edition. East Melbourne: RACGP; 2018 [cited 2019 May 21]. Available from: www.racgp.org.au/FSDEDEV/ media/documents/Clinical\%20Resources/Guidelines/ Red\%20Book/Guidelines-for-preventive-activities-ingeneral-practice.pdf

20. Cancer Council Australia Melanoma Guidelines Working Party. Clinical practice guidelines for the diagnosis and management of melanoma. Sydney: Cancer Council Australia; 2017 [cited 2019 May 21]. Available from: wiki.cancer.org.au/australia/Guidelines:Melanoma

21. Pashayan N, Morris S, Gilbert FJ, Pharoah PDP. Costeffectiveness and benefit-to-harm ratio of risk-stratified screening for breast cancer: a life-table model. JAMA Oncol. 2018;4:1504-10.

22. Cust AE, Aitken JF, Baade PD, Whiteman DC, Soyer HP, Janda M. Why a randomized melanoma screening trial may be a good idea. Br J Dermatol. 2018;179:1227-28.
23. Australian Skin and Skin Cancer Research Centre. Melanoma Screening Summit. Brisbane: ASSC; 2019 [cited 2019 May 28]. Available from: www.assc.org.au/ assc-melanoma-screening-summit-25-march-2019/

24. Lynch HT, de la Chapelle A. Hereditary colorectal cancer. NEJM. 2003;348(10):919-32.

25. Palomaki GE, McClain MR, Melillo S, Hampel HL, Thibodeau SN. EGAPP supplementary evidence review: DNA testing strategies aimed at reducing morbidity and mortality from Lynch syndrome. Genet Med. 2009;11(1):42-65.

26. Cancer Council Australia Colorectal Cancer Guidelines Working Party. Clinical practice guidelines for the prevention, early detection and management of colorectal cancer. Sydney: Cancer Council Australia; 2019 [cited 2019 Jun 4]. Available from: wiki.cancer.org.au/australia/ Guidelines:Colorectal_cancer

27. Win AK, Jenkins MA, Dowty JG, Antoniou AC, Lee A, Giles GG, et al. Prevalence and penetrance of major genes and polygenes for colorectal cancer. Cancer Epidemiol Biomarkers Prev. 2017;26(3):404-12.

28. Morris S, Rice T, O'Neill S, Raets E, Fairbank B. Misdiagnosed, misunderstood and missing out: Lynch syndrome, Australia's untold health story. Brisbane: Lynch Syndrome Australia; 2017 [cited 2019 Jun 4]. Available from: Iynchsyndrome.org.au/wp-content/ uploads/2017/03/Lynch-Syndrome-Report.pdf

29. Manchanda R, Patel S, Gordeev VS, Antoniou AC, Smith S, Lee A, et al. Cost-effectiveness of populationbased BRCA1, BRCA2, RAD51C, RAD51D, BRIP1, PALB2 mutation testing in unselected general population women. J Natl Cancer Inst. 2018;110(7):714-25.

30. National Institute for Health and Care Excellence. Molecular testing strategies for Lynch syndrome in people with colorectal cancer. UK: NICE; 2017 [cited 2019 Jun 4]. Available from: www.nice.org.uk/guidance/dg27

31. Kang YJ, Killen J, Caruana M, Simms K, Taylor N, Frayling IM, et al. The effectiveness and the costeffectiveness of systematic testing for Lynch syndrome in incident colorectal cancer cases in Australia. Med J Aust. Forthcoming.

32. Wedden S, Miller K, Frayling IM, Thomas T, Chefani A, Hamblin A, et al. Colorectal cancer stratification in the routine clinical pathway: a District General Hospital experience. Appl Immunohistochem Mol Morphol. 2018; Jul 5:doi:10.1097/PAI.0000000000000631.

33. Australia Government: Ministers, Department of Health. New test for breast cancer as thousands to benefit from new Medicare listings. Canberra: Australian Government; 2017 [cited 2019 Jun 25]. Available from: beta.health. gov.au/ministers/the-hon-greg-hunt-mp/media/new-testfor-breast-cancer-as-thousands-to-benefit-from-newmedicare-listings 
34. Cocker F, Chien Yee K, Palmer AJ, de Graaff B. Increasing incidence and mortality related to liver cancer in Australia: time to turn the tide. Aust N Z J Public Health. 2019;43(3);267-73.

35. Australian Institute of Health and Welfare. Cancer data in Australia; Australian Cancer Incidence and Mortality (ACIM) books: liver cancer. Canberra: AlHW; 2018 [cited 2019 Jun 11]. Available from: www.aihw.gov.au/reports/ cancer/cancer-data-in-australia/acim-books

36. European Association for the Study of the Liver. EASL Clinical Practice Guidelines: management of hepatocellular carcinoma. J Hepatol. 2018;69(1):182-236.

37. Singal AG, Pillai A, Tiro J. Early detection, curative treatment, and survival rates for hepatocellular carcinoma surveillance in patients with cirrhosis: a meta-analysis. PLoS Med. 2014;11(4):e1001624.

38. Choi DT, Kum HC, Park S, Ohsfeldt RL, Shen Y, Parikh ND, et al. Hepatocellular carcinoma screening is associated with increased survival of patients with cirrhosis. Clin Gastroenterol Hepatol. 2019;17(5):976-87.

39. Cucchetti A, Trevisani F, Pecorelli A, Erroi V, Farinati F, Ciccarese $F$, et al. Estimation of lead-time bias and its impact on the outcome of surveillance for the early diagnosis of hepatocellular carcinoma. J Hepatol. 2014;61(2):333-41.

40. van Meer S, de Man RA, Coenraad MJ, Sprengers D, van Nieuwkerk KM, Klumpen HJ, et al. Surveillance for hepatocellular carcinoma is associated with increased survival: results from a large cohort in the Netherlands. J Hepatol. 2015;63(5):1156-63.

41. Zhang BH, Yang BH, Tang ZY. Randomized controlled trial of screening for hepatocellular carcinoma. J Cancer Res Oncol. 2004;130(7):417-22.

42. Chen JG, Parkin DM, Chen QG, Lu JH, Shen QJ, Zhang BC, et al. Screening for liver cancer: results of a randomised controlled trial in Qidong, China. J Med Screen. 2003;10(4):204-9.

43. Moon AM, Weiss NS, Beste LA, Su F, Ho SB, Jin GY, et al. No association between screening for hepatocellular carcinoma and reduced cancer-related mortality in patients with cirrhosis. Gastroenterol. 2018;155(4):1128-39.
44. Ji M, Liu Z, Chang ET, Yu X, Wu B, Deng L, et al. Mass screening for liver cancer: results from a demonstration screening project in Zhongshan City, China. Sci Rep. 2018;8(1):12787.

45. Tzartzeva K, Obi J, Rich NE, Parikh ND, Marrero JA, Yopp A, et al. Surveillance imaging and alpha fetoprotein for early detection of hepatocellular carcinoma in patients with cirrhosis: a meta-analysis. Gastroenterol. 2018;154(6):1706-18.

46. Goossens N, Bian CB, Hoshida Y. Tailored algorithms for hepatocellular carcinoma surveillance: is one-size-fits-all strategy outdated? Curr Hepatol Rep. 2017;16(1):64-71.

47. Hong T, J Gow PJ, Fink M, Dev A, Roberts SK, Nicoll A, et al. Surveillance improves survival of patients with hepatocellular carcinoma: a prospective populationbased study. Med J Aust. 2018;209(8):348-54.

48. Allard N, Cabrie T, Wheeler E, Richmond J, MacLachlan J, Emery J, et al. The challenge of liver cancer surveillance in general practice: do recall and reminder systems hold the answer? Aust Fam Physician. 2017;46(11):859-64.

49. Singal AG, Tiro JA, Murphy CC, Marrero JA, McCallister K, Fullington $\mathrm{H}$, et al. Mailed outreach invitations significantly improve HCC surveillance rates in patients with cirrhosis: a randomized clinical trial. Hepatol. 2019;69(1):121-30.

50. Prostate Cancer Foundation of Australia, Cancer Council Australia. PSA testing guidelines expert advisory panel. PSA testing and early management of test-detected prostate cancer: clinical practice guidelines. Sydney: Prostate Cancer Foundation of Australia, Cancer Council Australia; 2016 [cited 2019 May 21]. Available from: www. prostate.org.au/media/612113/PSA-TestingGuidelines.pdf

51. Pickles K, Carter SM, Rychetnik L, McCaffery K, Entwistle VA. General practitioners' experiences of, and responses to, uncertainty in prostate cancer screening: insights from a qualitative study. PLoS One. 2016;11(4):e0153299.

52. Shaughnessy AF Cosgrove L, Lexchin JR. The need to systematically evaluate clinical practice guidelines. J Am Board Fam Med. 2016;29:644-8.

\section{Copyright: (c)}

(C) 2019 Weber et al. This article is licensed under the Creative Commons Attribution-NonCommercial-ShareAlike 4.0 International Licence, which allows others to redistribute, adapt and share this work non-commercially provided they attribute the work and any adapted version of it is distributed under the same Creative Commons licence terms. See: www.creativecommons.org/licenses/by-nc-sa/4.0/ 